\title{
Rheological Properties of Polysaccharides from Longan (Dimocarpus longan Lour.) Fruit
}

\author{
Xingxun Liu, ${ }^{1}$ Yongyue Luo, ${ }^{2}$ Chunjie Zha, ${ }^{2}$ Sumei Zhou, ${ }^{1}$ Liya Liu, ${ }^{1}$ and Lei Zhao ${ }^{3}$ \\ ${ }^{1}$ Institute of Agro-Products Processing Science and Technology (IAPPST), Chinese Academy of Agricultural Science, \\ Beijing 100193, China \\ ${ }^{2}$ Chinese Agricultural Ministry Key Laboratory of Tropical Crop Product Processing, Agricultural Product Processing Research Institute, \\ Chinese Academy of Tropical Agricultural Sciences, Zhanjiang 524001, China \\ ${ }^{3}$ College of Food Science, South China Agricultural University, Guangzhou 510642, China
}

Correspondence should be addressed to Xingxun Liu; ytboy652@163.com and Yongyue Luo; lyy6226@hotmail.com

Received 26 January 2015; Revised 27 April 2015; Accepted 29 April 2015

Academic Editor: Cornelia Vasile

Copyright (c) 2015 Xingxun Liu et al. This is an open access article distributed under the Creative Commons Attribution License, which permits unrestricted use, distribution, and reproduction in any medium, provided the original work is properly cited.

Longan polysaccharide (LP) was extracted from longan (Dimocarpus longan Lour.) pulp. The composition and rheological properties were determined by chemical analysis and dynamic shear rheometer. The flow behavior and viscoelastic behavior of longan polysaccharide (LP) solution were investigated by steady shear and small amplitude oscillatory shear (SAOS) experiments, respectively. The result shows that the solution is a pseudoplastic flow in a range of shear rate $\left(1-100 \mathrm{~s}^{-1}\right)$. The rheological behavior of LP was influenced by cations such as $\mathrm{Na}^{+}$and $\mathrm{Ca}^{2+}$. With an increase of apparent viscosity, $\mathrm{G}^{\prime}$ and $\mathrm{G}^{\prime \prime}$ were accompanied by addition of $\mathrm{Na}^{+}$and $\mathrm{Ca}^{2+}$.

\section{Introduction}

Polysaccharides have attracted much attention due to their broad spectra of therapeutic properties and relatively low toxicity [1-3]. Polysaccharides can be divided into animal polysaccharides, plant polysaccharides, and microbial polysaccharides according to their different sources. Plant polysaccharides are widely distributed and are of higher content among all polysaccharides. Plant polysaccharides also show some bioactivities, such as antioxidation, immunomodulation, antitumor, and hypoglycemic activities, in development of therapeutic drugs and high value healthy food in modern science [4].

Longan (Dimocarpus longan Lour.) which belongs to the Sapindaceae family, mainly planted in the south of China $[5,6]$, was welcomed by consumers due to health benefits $[7,8]$. The fresh seed and pericarp of the fruit have been used as traditional Chinese medicines (TCMs) for several decades. Logan pulp and pericarp contain polysaccharides, which have a variety of bioactivities, such as antioxidation [9] and antitumor activities [10] and the activity of antiglycation and antityrosinase [11].
Studying the rheological properties is important to application of polysaccharides functional properties, the gelation processing can be gotten from rheological data, and processing parameters of polysaccharides may affect the jelly preparation [12]. There are many factors that affect the rheological behavior of polysaccharides. The inner factor is microstructure of polysaccharides, such as molecular weight, degree of branches, and functional groups of polysaccharides [13]. Furthermore, temperatures, concentration of polysaccharide, and presence of sugars or salts also have an effect on rheological properties of polysaccharide [14-17]. Previous works on rheological properties of mango polysaccharide [18], psyllium polysaccharide [19], and polysaccharide from boat-fruited sterculia seeds [20], which are affected by factors such as the concentration of polysaccharide, cosolute, and $\mathrm{pH}$ conditions, were studied. To the best of our knowledge, there is no paper on rheological properties study on longan polysaccharides.

To the best of our knowledge, there is no paper to report the rheological properties of longan polysaccharide, so the aim of this work is to study the rheological properties of longan polysaccharides for further application. In order to 
prepare the polysaccharides, we used ethanol precipitate to prepare water-soluble polysaccharides. The composition and rheological properties were determined by chemical analysis and dynamic shear rheometer.

\section{Materials and Methods}

2.1. Plant Materials and Sample Preparation. The hot air dried Shixia longan (Dimocarpus longan Lour., harvested from Guangdong Province) pulp was soaked into fourfold $80 \%$ $(\mathrm{v} / \mathrm{v})$ ethanol for 48 hours to remove pigment and then dried before grinding into 200-mesh powder which was subject to further analysis.

2.2. Polysaccharide Extraction. Longan polysaccharide (LP) was extracted by hot water and alcohol precipitation according to Yi's methods [21]. In brief, $10 \mathrm{~g}$ of longan fruit pericarp powder was dispersed in $100 \mathrm{~mL}$ of distilled water and heated to $90^{\circ} \mathrm{C}$ for two hours once for three times. The extract was filtered through fourfold gauze and then centrifuged at $3000 \mathrm{rpm}$ for 10 minutes. The supernatant was concentrated to $100 \mathrm{~mL}$ using rotatory evaporator (LR4002, Heidolph, Germany) at $65^{\circ} \mathrm{C}$ under vacuum. Anhydrate ethanol $(400 \mathrm{~mL})$ was added to the concentration extract to precipitate polysaccharides overnight at $4^{\circ} \mathrm{C}$. The pellet was gathered after centrifugation, successively washed by anhydrate ethanol, absolute ether, and acetone, and then dried at $45^{\circ} \mathrm{C}$ under vacuum. The content of neutral polysaccharide was determined by the phenol-sulphuric acid method and expressed as glucose equivalents. The content of hexuronic acid was determined by the method of Blumenkrantz and Asboe-Hansen and expressed as glucuronic acid equivalents. The protein content was estimated using Folin-Ciocalteu's reagent and expressed as bovine serum albumin equivalents [8].

\subsection{Rheological Measurement}

2.3.1. Preparation of Samples. Water-soluble polysaccharide solutions at different concentrations $(0.5 \%, 3.0 \%, 5.0 \%$, and $10 \%, \mathrm{w} / \mathrm{v})$ were prepared by dissolving the dried polysaccharide powder in distilled water and samples $(5 \%, \mathrm{w} / \mathrm{v})$ dissolved in different $\mathrm{NaCl}$ concentration solutions $(0 \mathrm{mM}$, $50 \mathrm{mM}, 150 \mathrm{mM}$, and $250 \mathrm{mM}$ ) and different $\mathrm{CaCl}_{2}$ concentration solutions ( $0 \mathrm{mM}, 50 \mathrm{mM}, 150 \mathrm{mM}$, and $250 \mathrm{mM})$. All the samples were stirred for $2 \mathrm{~h}$.

2.3.2. Rheological Experiment. Dynamic shear rheological properties such as storage modulus $\left(G^{\prime}\right)$, loss modulus $\left(G^{\prime \prime}\right)$, and complex viscosity $\left(\eta^{*}\right)$ of the gels were measured under low-amplitude oscillatory shear using plate-and-plate geometry with diameter of $50 \mathrm{~mm}$ in an MCR Rheometer (Anton Paar, Austria, DE). The dynamic measurements were performed at a strain value of $0.02(2 \%)$ (within the linear viscoelastic region). Frequency sweep tests of all the solutions were performed using plate/plate geometry $(50 \mathrm{~mm}$ diameter, $0.5 \mathrm{~mm}$ gap) at $25^{\circ} \mathrm{C}$ and frequency $(\omega)$ from 0.1 to $100 \mathrm{~s}^{-1}$ to assess the flow behavior of the polysaccharide dispersions.

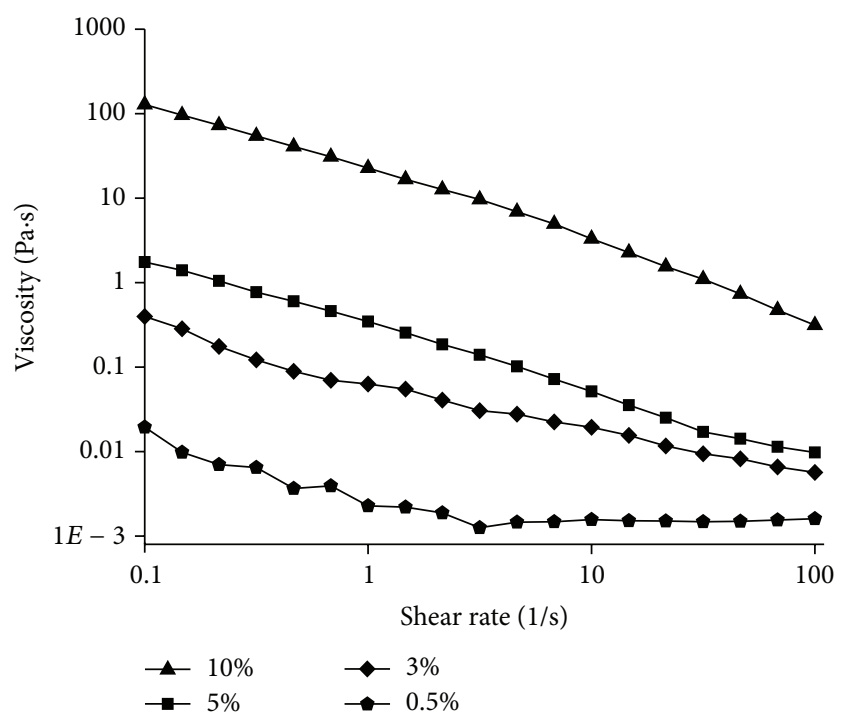

FIGURE 1: Steady shear flow curves of different concentration of LP.

\section{Results and Discussion}

The water-soluble polysaccharides were extracted from longan pulp by hot water and alcohol precipitation in this work. The longan polysaccharide (LP) was determined to contain $80.72 \%$ of polysaccharides, $5.27 \%$ of uronic acids, and $13.10 \%$ of proteins, which is similar to previous report [22].

3.1. The Viscosity Change of LP Solution. Studying the viscosity at the low shear rate could permit testing the consistency of the product in the mouth [23] while the viscosity at the high shear rate could provide some information of the product in processing operation, such as mixing and spray drying. In this paper, the effect of concentration on apparent viscosity for $0.5 \%, 3.0 \%, 5.0 \%$, and $10.0 \%(\mathrm{w} / \mathrm{v})$ crude longan polysaccharide solutions is shown in Figure 1. The apparent viscosity of each concentration solution obviously decreased with the increase of shear rate, showing a shear-thinning behavior, which means that this kind of crude longan polysaccharide solution belongs to non-Newtonian fluid (or pseudoplastic flow behavior). From Figure 1, it also could be found that the viscosity increased with increasing LP concentration; however, at lower concentrations $(0.5 \%)$, the rheological measurements became erratic. The shear-thinning phenomenon could be due to the rate of formation of new entanglements lower than externally imposed disruption rate with increasing in shear rate.

The effect of $\mathrm{Na}^{+}$and $\mathrm{Ca}^{2+}$ on the flow properties of LP was illustrated in Figures 2 and 3. With progressive addition of $\mathrm{Na}^{+}$solution, the viscosity of LP increased little at first $(50 \mathrm{mmol} / \mathrm{L})$ and then increased quickly after addition of $\mathrm{Na}^{+}$ $(100 \mathrm{mmol} / \mathrm{L})$. For $\mathrm{Ca}^{2+}$, the viscosity increased rapidly after addition of $\mathrm{CaCl}_{2}(50 \mathrm{mmol} / \mathrm{L})$ in Figure 3.

3.2. Cox-Merz Inconformity. The Cox-Merz rule (1) allows us to predict $\eta(\dot{\gamma})$ from oscillatory measurements when $\eta(\dot{\gamma})$ is difficult to measure at high shear rates because of sample 


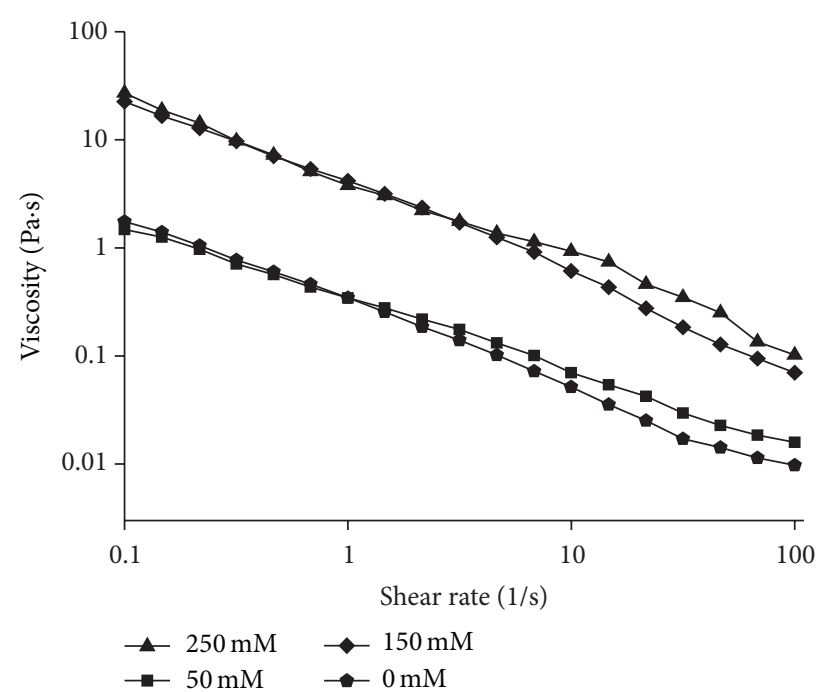

FIGURE 2: Steady shear flow curves of LP with different concentration of $\mathrm{Na}^{+}$.

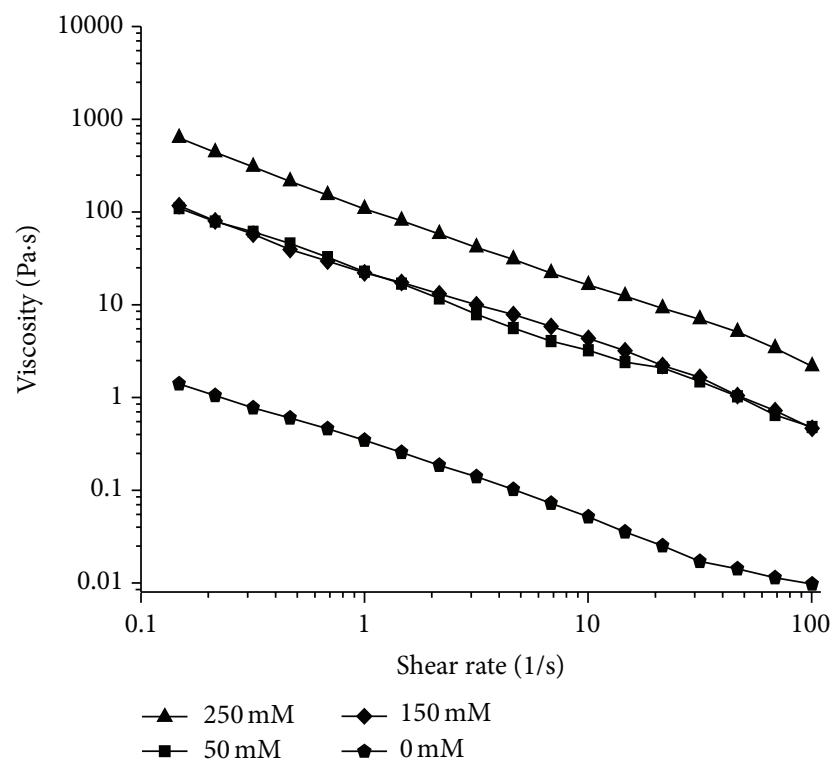

FIGURE 3: Steady shear flow curves of 5\% LP with different concentration of $\mathrm{Ca}^{2+}$.

fracture, secondary flows, and so forth, or we can estimate $\eta^{*}(\omega)$ from steady state viscosity data when oscillatory mode is not available. Another important application of the CoxMerz rule is to learn about the microstructure of materials from the degree of conformity with the rule. Therefore, the shear rate dependence of steady shear viscosity and the frequency dependence of complex viscosity were compared to check if LP sample conforms to the Cox-Merz rule. Steady shear viscosity is defined as $\eta=\tau / \dot{\gamma}$ in which $\tau$ stands for steady shear stress and $\dot{\gamma}$ for steady shear rate, and complex viscosity is defined as $\eta^{*}=\sigma / \gamma \omega=G^{*} / \omega=$ $\sqrt{G^{\prime 2}+G^{\prime \prime 2}} / \omega$, in which $\sigma$ stands for stress, $\gamma$ for strain, and $\omega$ for frequency. According to the result illustrated in

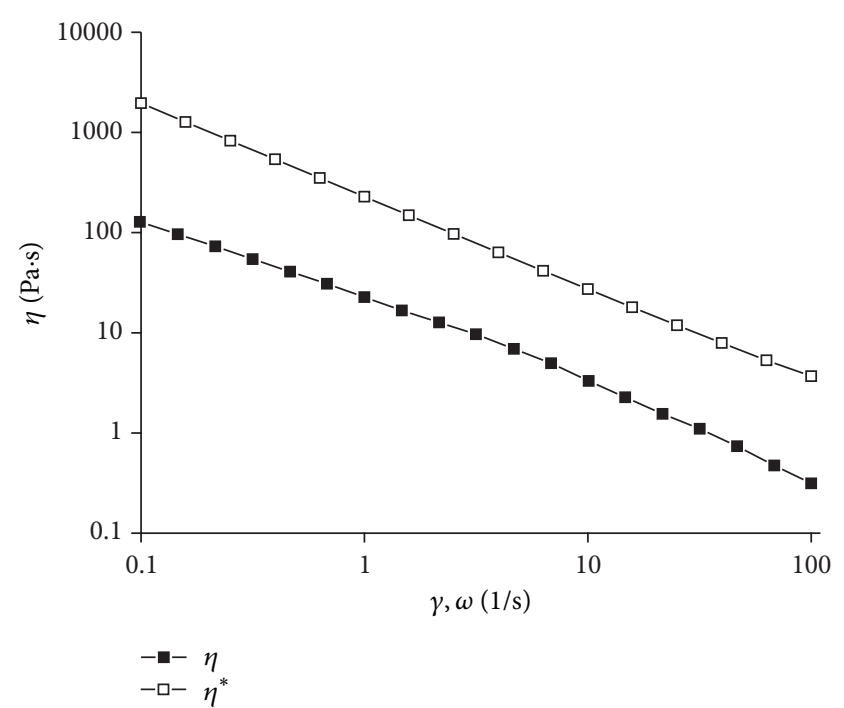

FIgUre 4: Cox-Merz plot of polysaccharide of $10 \%$ solution at $25^{\circ} \mathrm{C}$ $\left(\diamond-\eta ; \mathbf{\square}-\eta^{*}\right)$.

Figure 4, the longan polysaccharide solution did not meet Cox-Merz plot as shear viscosity differed in complex dynamic viscosity. This result indicated that it is not appropriate to estimate dynamic viscosity through apparent viscosity for this system, and vice versa. The inconformity of the CoxMerz rule implies that a network can be broken down during steady shear measurements but remains intact under small amplitude oscillation:

$$
\eta(\dot{\gamma})=\left.\eta^{*}(\omega)\right|_{\omega=\dot{\gamma}}
$$

3.3. Viscoelastic Properties. The viscoelastic properties of LP were evaluated by SAOS measurements. The frequency dependence of storage modulus $\left(G^{\prime}\right)$ and loss modulus $\left(G^{\prime \prime}\right)$ of longan polysaccharide solution with different concentration could be seen in Figure 5. A viscoelastic solid-like behavior with $G^{\prime}$ higher than $G^{\prime \prime}$ over the entire range of $\omega$ is clearly seen. Furthermore, both $G^{\prime}$ and $G^{\prime \prime}$ nearly keep a constant during the whole experiment, which means that both $G^{\prime}$ and $G^{\prime \prime}$ exhibit a little dependence of frequency. This phenomenon means that the LP showed typical "weak gel" structure.

The effect of $\mathrm{Na}^{+}$and $\mathrm{Ca}^{2+}$ on viscoelastic properties of LP was measured and the result could be found in Figures 6 and 7. Immediate sharp increase in $G^{\prime}$ and $G^{\prime \prime}$ (see Figure 4) on progressive addition of $\mathrm{NaCl}$ or $\mathrm{CaCl}_{2}$ to $5 \% \mathrm{LP}$, with more gradual increase at $\mathrm{Na}^{+}$or $\mathrm{Ca}^{2+}$ concentrations above $\sim 50 \mathrm{mM}$, was seen. However, the $\mathrm{Ca}^{2+}$ has a more significant effect on $G^{\prime}$ and $G^{\prime \prime}$ than $\mathrm{Na}^{+}$. Effect of salt on LP is broadly similar to that of gellan, which is anionic and forms double helices [24]. For gellan, monovalent (Group I) metal ions promote gelation by binding to individual double helices, thus suppressing the electrostatic barrier to helix-helix association. Binding occurs only above a minimum critical cation concentration, which for $\mathrm{Na}^{+}$is $\sim 65 \mathrm{mM}$ (the concentration at which the moduli and viscosity of LP increase steeply on 


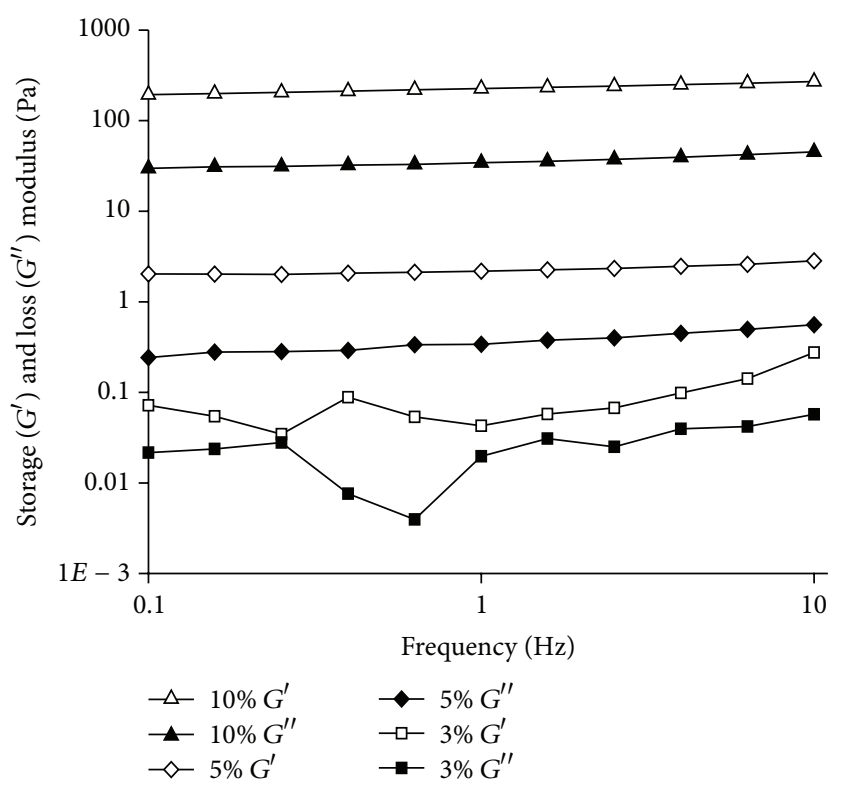

Figure 5: Frequency dependence of storage $\left(G^{\prime}\right)$ and loss $\left(G^{\prime \prime}\right)$ modulus of different concentration of LP.

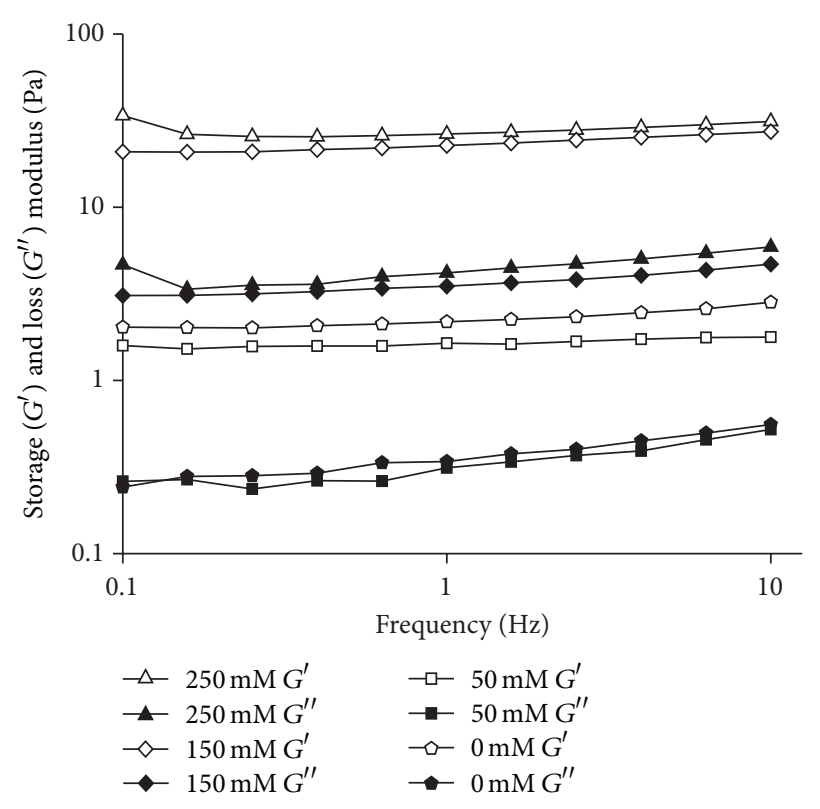

Figure 6: Frequency dependence of storage $\left(G^{\prime}\right)$ and loss $\left(G^{\prime \prime}\right)$ modulus of $5 \%$ LP with different content of $\mathrm{Na}^{+}$.

progressive addition of $\mathrm{NaCl}$ ). $\mathrm{Ca}^{2+}$ promotes gelation by binding between helices, and there is an immediate sharp increase in moduli on progressive addition of $\mathrm{CaCl}_{2}$ to gellan solutions (as seems also to occur with LP). These apparent similarities may be coincidental, but the effect of salts on LP in greater detail would be worth exploring, and the details will be studied in the future.

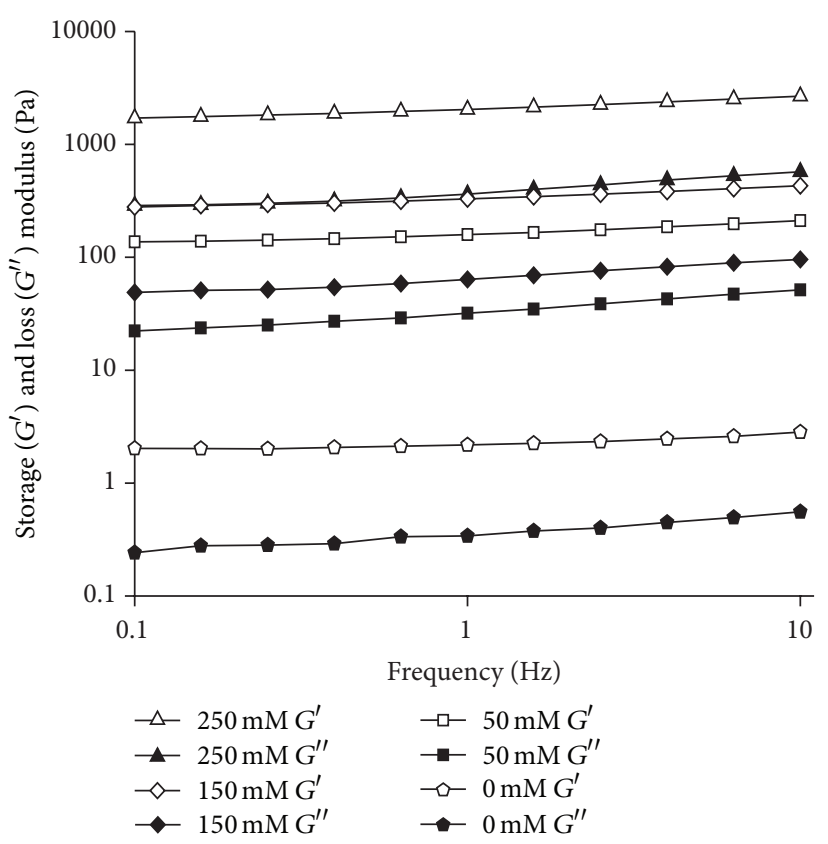

FIGURE 7: Frequency dependence of storage $\left(G^{\prime}\right)$ and loss $\left(G^{\prime \prime}\right)$ modulus of $5 \%$ LP with different content of $\mathrm{Ca}^{2+}$.

\section{Conclusion}

The rheological properties of water-soluble polysaccharides from longan (Dimocarpus longan Lour.) fruit were studied in this paper. Steady shear rheological measurements showed a non-Newtonian shear-thinning flow behavior and it disconforms to Cox-Merz empirical correlation. Both fluidlike $\left(G^{\prime \prime}\right.$ dominant) and solid-like ( $G^{\prime}$ dominant) behaviors were observed when a cosolute was added or acidic condition changed. It was found that the addition of $\mathrm{Na}^{+}$and $\mathrm{Ca}^{2+}$ had influence on viscosity and fluidlike or solid-like behaviors of longan polysaccharide. The current paper provided a preliminary exploration of rheological properties of longan polysaccharide.

\section{Conflict of Interests}

The authors declare that there is no conflict of interests regarding the publication of this paper.

\section{Acknowledgments}

This work was financially supported by NSFC (31301554) and the national tropical crops engineering development of Reconstruction Project of Chinese National Engineering Research Center (no. 2011FU125Z09).

\section{References}

[1] B. Yang, M. Zhao, J. Shi, G. Cheng, N. Ruenroengklin, and Y. Jiang, "Variations in water-soluble saccharides and phenols in longan fruit pericarp after drying," Journal of Food Process Engineering, vol. 31, no. 1, pp. 66-77, 2008. 
[2] Y.-C. Cheung, X.-X. Liu, W.-Q. Wang, and J.-Y. Wu, "Ultrasonic disruption of fungal mycelia for efficient recovery of polysaccharide-protein complexes from viscous fermentation broth of a medicinal fungus," Ultrasonics Sonochemistry, vol. 22, pp. 243-248, 2014.

[3] S. Chen, K.-C. Siu, W.-Q. Wang, X.-X. Liu, and J.-Y. Wu, "Structure and antioxidant activity of a novel poly-N-Acetylhexosamine produced by a medicinal fungus," Carbohydrate Polymers, vol. 94, no. 1, pp. 332-338, 2013.

[4] B. Yang, Y. Jiang, R. Wang, M. Zhao, and J. Sun, "Ultra-high pressure treatment effects on polysaccharides and lignins of longan fruit pericarp," Food Chemistry, vol. 112, no. 2, pp. 428431, 2009.

[5] Y. Pan, K. Wang, S. Huang et al., "Antioxidant activity of microwave-assisted extract of longan (Dimocarpus Longan Lour.) peel," Food Chemistry, vol. 106, no. 3, pp. 1264-1270, 2008.

[6] G. Jiang, L. Wen, F. Chen et al., "Structural characteristics and antioxidant activities of polysaccharides from longan seed," Carbohydrate Polymers, vol. 92, no. 1, pp. 758-764, 2013.

[7] B. Yang, M. Zhao, K. N. Prasad, G. Jiang, and Y. Jiang, "Effect of methylation on the structure and radical scavenging activity of polysaccharides from longan (Dimocarpus longan Lour.) fruit pericarp," Food Chemistry, vol. 118, no. 2, pp. 364-368, 2010.

[8] Y. Yi, S.-T. Liao, M.-W. Zhang et al., "Immunomodulatory activity of polysaccharide-protein complex of longan (dimocarpus longan lour.) pulp," Molecules, vol. 16, no. 12, pp. 10324-10336, 2011.

[9] B. Yang, Y. Jiang, M. Zhao et al., "Structural characterisation of polysaccharides purified from longan (Dimocarpus longan Lour.) fruit pericarp," Food Chemistry, vol. 115, no. 2, pp. 609$614,2009$.

[10] K. Zhong, Q. Wang, Y. He, and X. He, "Evaluation of radicals scavenging, immunity-modulatory and antitumor activities of longan polysaccharides with ultrasonic extraction on in S180 tumor mice models," International Journal of Biological Macromolecules, vol. 47, no. 3, pp. 356-360, 2010.

[11] B. Yang, M. Zhao, and Y. Jiang, "Anti-glycated activity of polysaccharides of longan (Dimocarpus longan Lour.) fruit pericarp treated by ultrasonic wave," Food Chemistry, vol. 114, no. 2, pp. 629-633, 2009.

[12] H. Wang, X. Zhang, Y. Li et al., "Antitumor activity of a polysaccharide from longan seed on lung cancer cell line A549 in vitro and in vivo," Tumor Biology, vol. 35, no. 7, pp. 7259-7266, 2014.

[13] Y. Tao and D. Feng, "Dilute solution and rheological properties of hyperbranched polysaccharide from Pleurotus tuber-regium sclerotia," Food Hydrocolloids, vol. 28, no. 1, pp. 151-158, 2012.

[14] M. T. Nickerson and A. T. Paulson, "Rheological properties of gellan, $\kappa$-carrageenan and alginate polysaccharides: effect of potassium and calcium ions on macrostructure assemblages," Carbohydrate Polymers, vol. 58, no. 1, pp. 15-24, 2004.

[15] M. T. Nickerson, A. T. Paulson, and F. R. Hallett, "Pre-gel solution properties of gellan polysaccharides: effect of potassium and calcium ions on chain associations," Food Research International, vol. 41, no. 5, pp. 462-471, 2008.

[16] M. T. Nickerson, A. T. Paulson, and R. A. Speers, "Rheological properties of gellan solutions: effect of calcium ions and temperature on pre-gel formation," Food Hydrocolloids, vol. 17, no. 5, pp. 577-583, 2003.

[17] C. Karakasyan, S. Lack, F. Brunel, P. Maingault, and D. Hourdet, "Synthesis and rheological properties of responsive thickeners based on polysaccharide architectures," Biomacromolecules, vol. 9, no. 9, pp. 2419-2429, 2008.
[18] F. Iagher, F. Reicher, and J. L. M. S. Ganter, "Structural and rheological properties of polysaccharides from mango (Mangifera indica L.) pulp," International Journal of Biological Macromolecules, vol. 31, no. 1-3, pp. 9-17, 2002.

[19] Q. Guo, S. W. Cui, Q. Wang, H. D. Goff, and A. Smith, "Microstructure and rheological properties of psyllium polysaccharide gel," Food Hydrocolloids, vol. 23, no. 6, pp. 1542-1547, 2009.

[20] Y. Wu, S. W. Cui, J. Wu, L. Ai, Q. Wang, and J. Tang, "Structure characteristics and rheological properties of acidic polysaccharide from boat-fruited sterculia seeds," Carbohydrate Polymers, vol. 88, no. 3, pp. 926-930, 2012.

[21] Y. Yi, S. T. Liao, M. W. Zhang et al., "Physicochemical characteristics and immunomodulatory activities of three polysaccharide-protein complexes of longan pulp," Molecules, vol. 16, no. 7, pp. 6148-6164, 2011.

[22] Y. Yi, M.-W. Zhang, S.-T. Liao et al., "Structural features and immunomodulatory activities of polysaccharides of longan pulp," Carbohydrate Polymers, vol. 87, no. 1, pp. 636-643, 2012.

[23] E. R. Morris and L. J. Taylor, "Oral perception of fluid viscosity," Progress in Food and Nutrition Science, vol. 6, pp. 285-296, 1982.

[24] E. R. Morris, K. Nishinari, and M. Rinaudo, "Gelation of gellan-a review," Food Hydrocolloids, vol. 28, no. 2, pp. 373-411, 2012. 

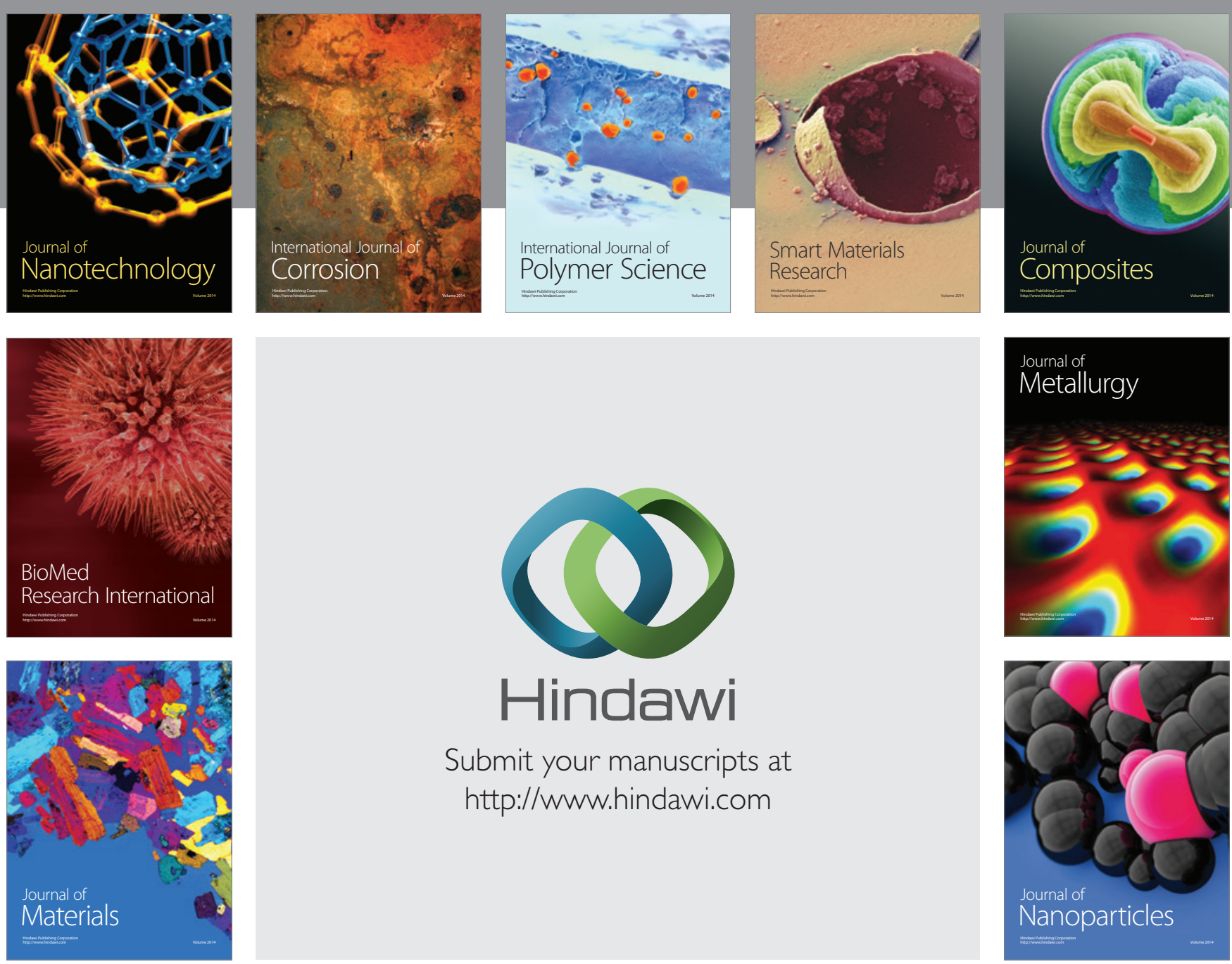

Submit your manuscripts at http://www.hindawi.com
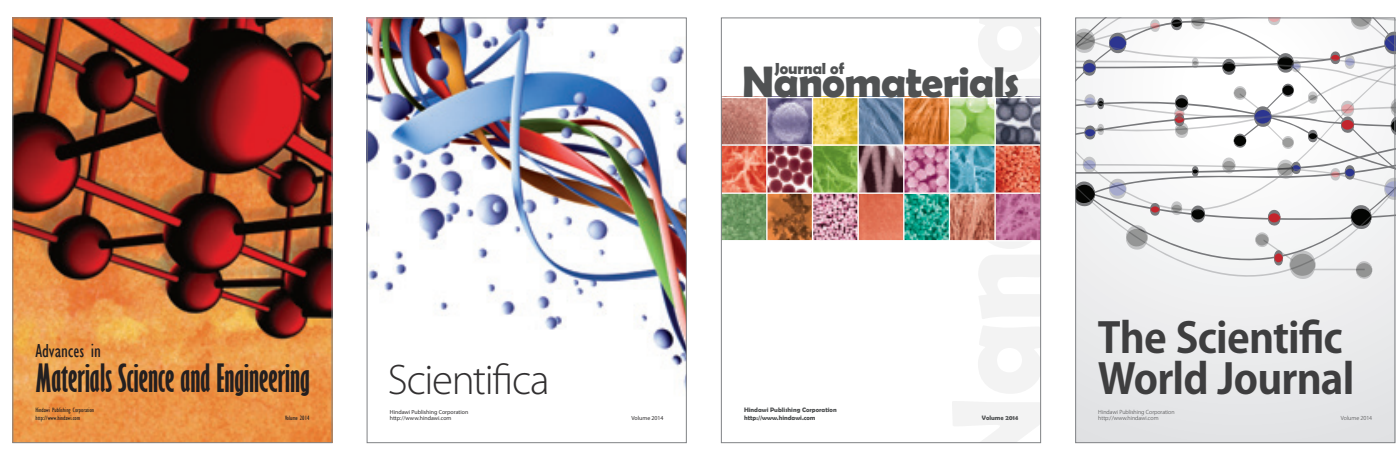

\section{The Scientific World Journal}
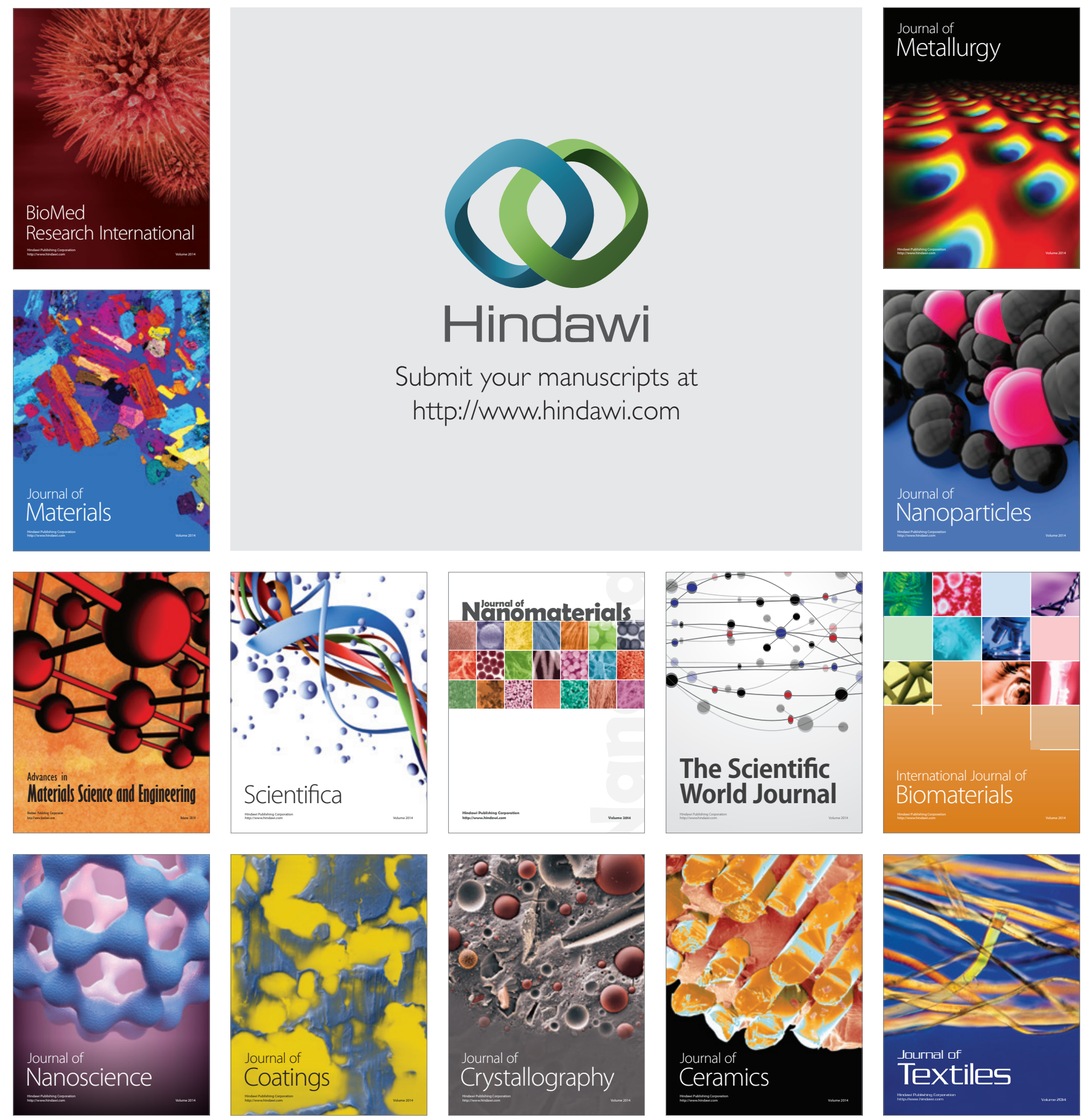